\title{
Nivel de ruido en la unidad de cuidado intensivo adulto: Medición, estándares internacionales e implicancias sanitarias
}

Noise level in the intensive care unit for adults: measurement, international standards and health implications

\author{
Angélica Patricia Garrido-Galindo', Yiniva Camargo-Caicedo², Andrés M. Vélez-Pereira ${ }^{3}$
}

1 Ingeniera Ambiental y Sanitaria, Estudiante de Maestría en Ingeniería Ambiental, Grupo de Investigación en Modelación de Sistemas Ambientales GIMSA, Universidad del Magdalena. Santa Marta Colombia. e-mail: garridogap@gmail.com

2 Ingeiera Química, Máster en Educación Ambiental. Docente-Investigadora, Grupo de Investigación en Modelación de Sistemas Ambientales GIMSA, Universidad del Magdalena. Santa Marta, Colombia.

e-mail: ycamargo@unimagdalena.edu.co

3 Ingeniero Ambiental y Sanitario, Magíster en Tecnología Ambiental, Estudiante de Doctorado en Ciencia y Tecnología Ambientales. Grupo de Investigación en Modelación de Sistemas Ambientales GIMSA, Universidad del Magdalena. Santa Marta, Colombia. e-mail: ing.andresvelez@gmail.com

\section{Resumen}

Objetivo: Evaluar el nivel continuo equivalente de ruido en una unidad de cuidado intensivo adulto perteneciente a un hospital de la ciudad de Santa Marta con los niveles sugeridos por los estándares internacionales. Materiales y métodos: Se realizó un muestreo continuo durante 20 días registrando datos cada minuto con un sonómetro tipo I en un eje central de la unidad de cuidado intensivo adulto del hospital; los datos registrados correspondían a los valores máximos, mínimos y el nivel continuo equivalente de ruido en ponderación frecuencial A y el filtro de ponderación temporal Fast. Resultados: Se reporta que el nivel de ruido continuo equivalente varió entre 49 - 71 dBA con un valor máximo de 91,19 dBA y un mínimo de 46,65 dBA. El análisis de varianza infiere que el valor medio de ruido varía con la hora del día. Conclusiones: El nivel de ruido continuo equivalente presentado en la unidad de cuidado intensivo adulto es consistente con otros estudios similares, no obstante los valores presentados exceden los estándares internacionales de ruido sugeridos, generando una preocupación por los riesgos de exposición a los que se encuentran pacientes y personal médico de la unidad.

Palabras clave: Monitoreo del ruido, salud pública, ruido en el ambiente de trabajo, cuidados intensivos. (Fuente: DeCS, Bireme).

\begin{abstract}
Objective: To assess the continuous equivalent noise level in an intensive care unit for adults that belongs to a hospital in the city of Santa Marta using the levels suggested by international standards. Materials and methods: A continuous sampling was done during 20 days where the data was recorded every minute by using a sound level meter type I in a central axis of the intensive care unit for adults in the hospital; the recorded data corresponded to the maximum, minimum values and the equivalent continuous level of noise on frequency weighting A and the Fast time weighting filter. Results: It was reported that the equivalent continuous noise level varied between 49-71 dBA with a maximum value of 91, $19 \mathrm{dBA}$ and a minimum of 46, $65 \mathrm{dBA}$. The variance analysis infers that the mean value of noise varies according to the time of day. Conclusions: The level of equivalent continuous noise presented in the intensive care unit for adults is consistent with other similar studies; however, the displayed values exceed the international standards of noise suggested, which generates a concern about the risks of exposure for the patients as well as for the medical staff of the unit.
\end{abstract}

Keywords: Noise monitoring, public health, noise in the working environment, intensive care. (Source: DeCS, Bireme). 


\section{Introducción}

El ambiente dentro de las unidades de cuidado intensivo (UCI) está influenciado por múltiples factores estresantes para los pacientes y para el personal que labora en éstas. Es así como el nivel de ruido se establece como uno de los agentes que deterioran el nivel de confort en la unidad. Los principales efectos sobre los pacientes asociados a la exposición a ruido excesivo dentro de las UCI son el aumento de la frecuencia cardíaca y tensión arterial, la privación de sueño y alteraciones en la función inmunológica. Adicionalmente el ruido excesivo incide sobre el bienestar del personal asistencial que labora en la unidad de cuidado crítico causando estrés y contribuyendo a la sensación de irritabilidad y fatiga. ${ }_{1,2}$

Los efectos asociados a niveles de ruido excesivos en unidades de cuidado crítico varían según la edad del paciente, en adultos es frecuente la privación del sueño que repercute de forma negativa en aspectos físicos, psicológicos y de conducta, retrasando con ello en muchos casos el proceso de recuperación de la enfermedad; en niños pueden presentarse traumas acústicos, respuestas de estrés fisiológico, privación del sueño, confusión y desorientación; por último en neonatos puede influenciar la aparición de problemas clínicos como apnea, bradicardia, deficiencias nutricionales, cambios en la percepción del sonido, pérdida de audición y alteraciones en el crecimiento y desarrollo normal del sistema nervioso central, mientras que en el personal de salud favorece la aparición del síndrome Burnout. ${ }_{1,3-12}$

A nivel normativo se resaltan en la temática de niveles de ruido estándares internacionales como los de la EPA (45 dBA en el día y $35 \mathrm{dBA}$ en la noche), el Consejo Internacional de Ruido - INC (45 dBA día y 20 dBA noche), el Instituto Nacional para la Seguridad y Salud Ocupacional - NIOSH (40 dBA día y 35 dBA noche), la OMS (35 dBA día y 30dBA noche) y el Real Decreto Ley 1386 de 1989 - RDL-1386 (40 dBA día y 35 dBA noche) citados por varios autores ${ }_{1,13-17}$

A nivel nacional la Resolución 8321 del 4 de Agosto de 1983 - R-8321 (aplicable para mediciones en interiores), dicta normas sobre la protección y conservación de la audición de la salud y el bienestar de las personas por causa de la producción y emisión de ruidos, especificando para zonas de tranquilidad niveles de presión sonora de $45 \mathrm{dBA}$ para el período diurno y nocturno. ${ }_{18}$

Por otro lado, la Resolución 627 del 7 de abril de 2006 - R-627 (aplicable para mediciones en ambientes exteriores) establece la norma nacional de emisión de ruido y ruido ambiental señalando como estándares máximos permisibles de niveles de emisión de ruido para el Sector A tranquilidad y silencio, valores de 55 para el día y de 50 para la noche. ${ }_{19}$

Para las unidades de cuidado intensivo adulto se reportan niveles promedio de $65 \mathrm{dBA}$, Elliott et al., en su estudio registran valores comprendidos entre $48 \mathrm{dBA}$ - $75 \mathrm{dBA}_{{ }_{20}}$ de la misma manera en Maracay (Venezuela) se encontraron niveles entre $66 \mathrm{dBA}-70$ $\mathrm{dBA}$, en Michigan (Estados Unidos) registros entre 60 dBA - 72 dBA y en Sao Paulo (Brasil), resultados entre 58,9 dBA - 64,1 dBA para el LA ${ }_{\text {eq }}{ }^{* 16,20-22}$

Por lo anterior, el propósito del presente estudio es evaluar el nivel continuo equivalente de ruido en una unidad de cuidado intensivo adulto perteneciente a un hospital de la ciudad de Santa Marta con los niveles sugeridos por los estándares internacionales, puesto que a nivel local se adolece de un estudio que permita verificar los niveles actuales de ruido manejados por estas unidades de cuidado crítico.

\section{Materiales y métodos}

\section{Área de estudio}

El estudio se desarrolló en la Unidad de Cuidado Intensivo Adulto (UCIA) de un hospital ubicado en la ciudad de Santa Marta (Colombia). La unidad cuenta con 19 camas habilitadas para el cuidado crítico. El hospital es un centro de atención médica de alta complejidad en la región, de carácter público que presta sus servicios médicos, al tiempo que se establece como escenario para la docencia universitaria en el área de la salud. En la UCIA laboran por turno 7 miembros del equipo asistencial, y la ocupación promedio de camas en la unidad es de $52,6 \%$ (10 camas), siendo este el número promedio de pacientes presentes en la UCIA. ${ }_{23}$

A lo largo de un día en la UCIA se desarrollan tres turnos del personal asistencial (mañana: 7:01-13:00, vespertina: 13:01-19:00 y noche: 19:01-7:00+1); 
así mismo, a las 8:00, 12:00 y 16:00 se brinda la alimentación al paciente. Finalmente, se establecen dos horarios de visita de familiares de 20 minutos, realizados a las 10:00 y las 17:00 $\mathrm{h}$.

\section{Diseño muestral}

El muestreo se realizó de forma continua durante 20 días en la UCIA entre los meses de agosto y septiembre de 2012, teniendo en cuenta la metodología expuesta en el estudio desarrollado por Vélez-Pereira, y FortesGarrido et al., que empleó un sonómetro tipo I marca Casella modelo CEL-633-C1K1 ${ }^{\circledR}$ y se programó para la toma de datos cada 60 segundos utilizando el filtro de ponderación frecuencial A y el filtro de ponderación temporal Fast. Dentro de los parámetros acústicos registrados se reportó el LAmín (para establecer el nivel de ruido mínimo) y el LAmax (para establecer los valores máximos) ${ }_{24,25}$

El sonómetro se ubicó teniendo en cuenta la metodología, la dinámica interna de la UCIA y recomendaciones de los coordinadores médicos. En la unidad se estableció un punto de muestreo ubicado en el ambiente a una distancia de $55 \mathrm{~cm}$ del techo y $123 \mathrm{~cm}$ de la pared en un lugar central.

\section{Procesamiento de la información}

Los datos obtenidos durante el muestreo realizado se procesaron inicialmente con la herramienta Microsoft Excel $^{\circledR}$ y posteriormente se ejecutó el análisis estadístico con el programa Statgraphics Centurion XVII para determinar si existe una influencia de la hora del día y el nivel de ruido.

\section{Nivel continuo equivalente}

En las hojas de cálculo trabajadas con Microsoft Excel ${ }^{\circledR}$ se generaron los gráficos de línea y se representaron los resultados de las mediciones obtenidas en la UCIA (LAeq, LAFMax y LAFMin); adicionalmente se representaron los datos correspondientes bajo un periodo de integración de 1 hora, lo que permitió analizar durante el tiempo de muestreo la variabilidad asociada a ciclos horarios o diarios. Para la obtención del promedio integrado se empleó la siguiente ecuación: ${ }_{24}$

$$
L A_{e q, 1 h}=10 * \log _{10}\left\lfloor\frac{\sum_{i=1}^{n} 10^{\frac{L e q i j}{10}}}{n_{j}}\right\rfloor
$$

Donde i corresponde a la observación del nivel continuo equivalente en ponderado A reportada en el intervalo de horario j, y $\mathrm{n}$ es el número total de observaciones i en la hora $\mathrm{j}$.

Con el paquete Statgraphics Centurion XVII se determinó el comportamiento estadístico de los niveles de ruido en la UCIA comparando las medias integradas horarias de LAeq de cada hora durante los 20 días de muestreo; para ello se elaboró un análisis de varianza - ANOVA que permitió establecer la existencia o inexistencia de diferencias estadísticamente significativas entre las medias horarias integradas del LAeq de los períodos evaluados en la unidad. ${ }_{24,26}$

\section{Resultados}

En el gráfico 1 se muestra el nivel continuo equivalente de ruido bajo el período de integración de 10 minutos en la UCIA, los resultados presentaron un comportamiento estable en los valores del $\mathrm{LA}_{\text {eq,10min }}$ registrados cada 24 horas, mostrando valores similares a las mismas horas (10:30 horas) y formando algunos grupos, del 19/08/2012 al 22/08/2012 con valores de $56 \mathrm{dBA}$ aproximadamente cada 24 horas; de la misma manera del 23/08/2012 al 24/08/2012 con valores de $57 \mathrm{dBA}$, del 29/08/2012 al 02/09/2012 con valores de 55 dBA y del 03/09/2012 al 07/09/2012 con valores de $58 \mathrm{dBA}$, lo que sugiere valores promedio de $57 \mathrm{dBA}$ aproximadamente a las 10:30 horas de cada día de muestreo en la UCIA.

Observando individualmente cada intervalo de 24 horas se detectaron descensos en los niveles de ruido en cada intervalo específicamente entre las 22:00 03:00 horas de cada intervalo diario. 
Gráfico 1. Nivel continuo equivalente de ruido en la UCIA

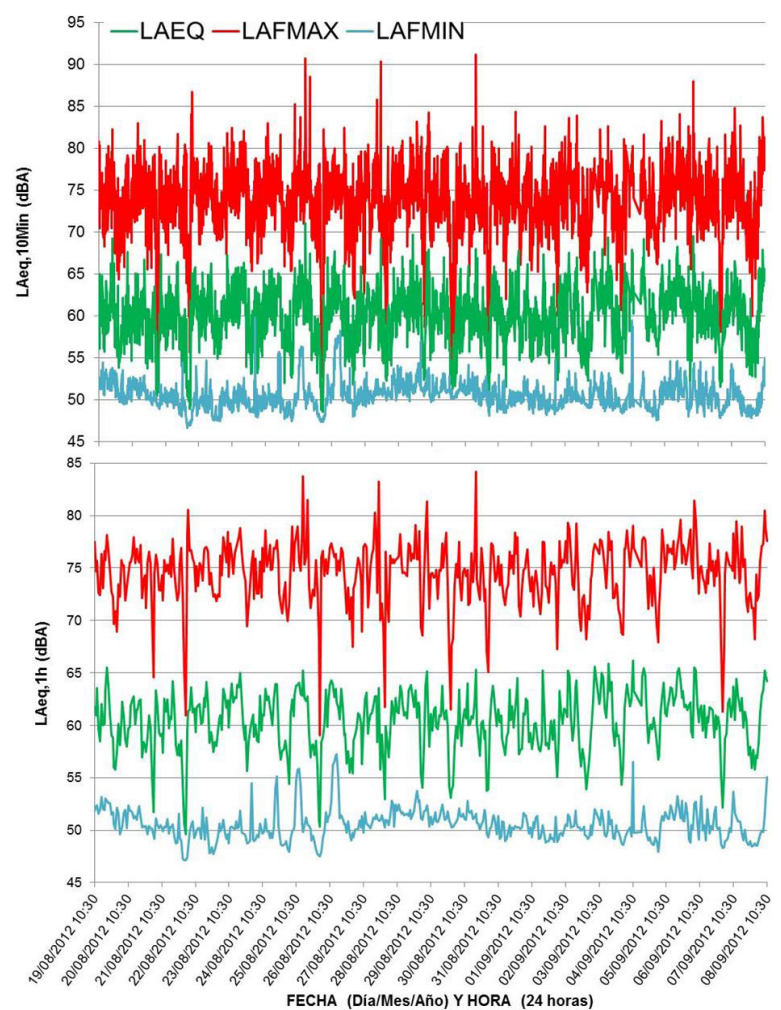

Los valores del $\mathrm{LA}_{\text {eq, } 10 \mathrm{~min}}$ presentan un rango de gran amplitud debido a que los registros se encontraron entre $49 \mathrm{dBA}$ - $71 \mathrm{dBA}$, por su parte el comportamiento de los valores máximos presentados en esta UCIA fue semejante al presentado por el LA $_{\text {eq,10min }}$ reportando valores de 90,73 dBA correspondiente al día 25/08/2012 a las 15:30 horas y de 91,19 dBA correspondiente al día 30/08/2012 a las 18:30 horas; este último, probablemente asociado al horario de entrega de turnos dentro de la unidad. Con relación a los mínimos se observa que el comportamiento se adapta a la tendencia mostrada por el $\mathrm{LA}_{\text {eq,10min, }}$ con valores de 46,65 dBA a las 02:20 horas y de 47,37 dBA a las 02:40 horas de los días 22/08/2012 y $26 / 08 / 2012$ respectivamente. Adicionalmente en el gráfico 1 se evidencian muy pocas diferencias entre el comportamiento de los días laborables o de mayor actividad (de lunes a viernes) y los fines de semana (sábados y domingos).
En cuanto a la variación horaria, los datos de la tabla 1 permiten establecer por el análisis de varianza que la hora del día tiene un incidencia significativa en el nivel de ruido continuo equivalente en ponderación A, existiendo una diferencia estadísticamente significativa entre las medias horarias, con un nivel del 95,0\% de confianza; mientras que el análisis ANOVA evidencia que no hay una diferencia significativa entre los días laborables y los fines de semana.

Tabla 1. Nivel continuo equivalente de ruido en la UCIA (dBA) y resultado ANOVA

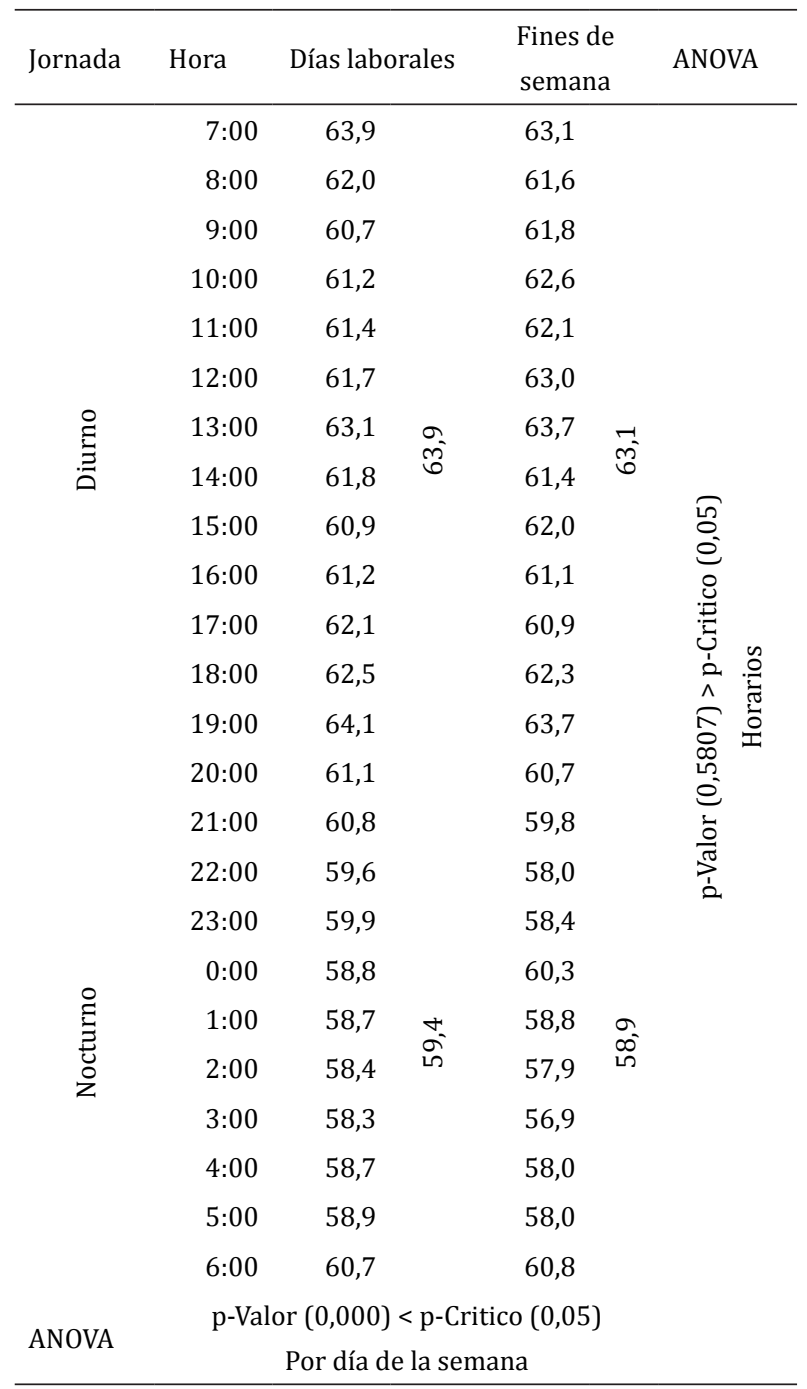




\section{Discusión}

Los resultados reportados por la unidad de cuidado intensivo adulto para el $\mathrm{LA}_{\mathrm{eq}}$ muestran claramente registros superiores a los sugeridos por la OMS; de hecho comparando los registros con estándares internacionales referenciados anteriormente se evidencia que difieren de las normas aplicadas en varios países, especialmente del límite de $20 \mathrm{dBA}$ durante la noche establecido por el INC y superado en la UCIA por casi el doble de lo establecido, puesto que en ninguna de las mediciones se obtuvo un valor cercano a $20 \mathrm{dBA}$, a diferencia del límite más alto fijado por estándares internacionales para período nocturno y que corresponde a $35 \mathrm{dBA}$ el cual es superado en menor medida.

Por otro lado, se encontró que los límites fijados para el horario diurno son más altos que los fijados para el horario nocturno, de igual manera si se comparan con los registros se observa que es superado en el $100 \%$ de las mediciones, pues en ninguno de los casos se obtuvieron valores por debajo de los $45 \mathrm{dBA}$ (Gráfico 2).

De igual manera, al contrastar los resultados obtenidos en la UCIA, con los límites aplicables a nivel nacional para ambientes interiores en zonas de tranquilidad como hospitales (45 dBA en el día y en la noche) se evidencia que el límite es superado en ambos períodos diurno y nocturno, incluso en los horarios correspondientes a la madrugada (registros más bajos), los valores reportados se alejan considerablemente de los niveles máximos permitidos por la normativa aplicable (Gráfico 2).

Aunque los LAeq, LAFmax y LAFmin registrados por el estudio son valores cercanos a los publicados por otros autores para ruido en las unidades de cuidado intensivo en al ámbito nacional e internacional, es preciso resaltar que estos niveles pueden llegar a tener implicaciones dentro de la unidad, en función a la respuesta a estímulos auditivos que depende de cada individuo, a la sensibilidad de cada paciente al ruido y a la presencia de patologías previas. ${ }_{1621,2227-29}$

Los efectos sobre los pacientes son producidos por la exposición persistente a altos niveles de ruido pueden repercutir en la estancia del paciente pues el proceso de recuperación es más lento, aumentando con ello el tiempo de permanencia en la unidad, e incrementando los costos de la estancia. ${ }_{30}$

\section{Gráfico 2. Nivel continuo equivalente de ruido promedio horario en la UCIA y estándares internacionales de ruido}

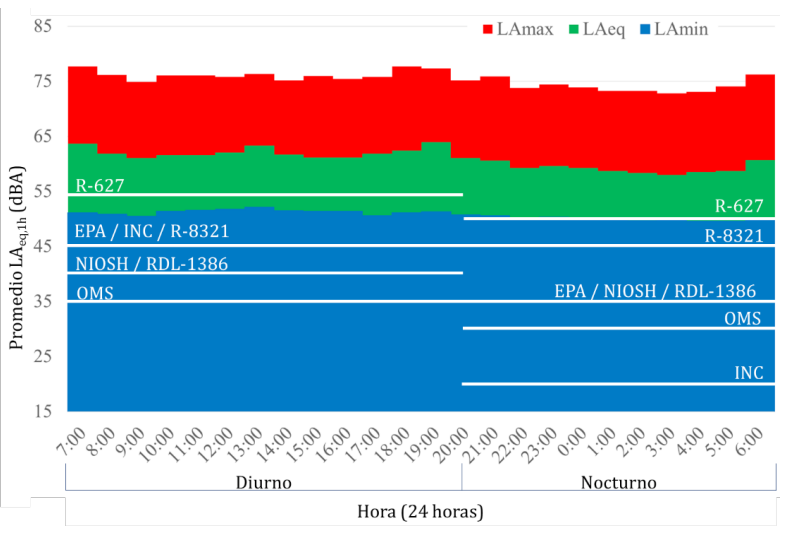

Estudios desarrollados en Colombia por AchurySaladaña y Achury, describen la influencia de variables como el ruido en el descanso del paciente, fuentes de ruido como los equipos biomédicos, el teléfono y las conversaciones del personal médico, alteran el ciclo del sueño-vigilia, disminuyendo con ello la calidad del sueño. ${ }_{31}$

Adicionalmente Fajardo et al., mencionan en su publicación la influencia del ruido en la recuperación del paciente internado; teniendo en cuenta que los resultados de las mediciones arrojaron valores por encima de los límites evaluados en su estudio (45 dBA día y 35 dBA noche), exponen la necesidad de implementar acciones que contribuyan a alcanzar niveles óptimos en las unidades de cuidado crítico $_{27,31}$

Por otro lado, la exposición constante por parte del personal médico, enfermeras y auxiliares de enfermería a estos niveles de ruido registrados en la UCIA objeto de estudio durante el tiempo laboral, incrementa el riesgo de causar desgaste en el personal, disminución del rendimiento e incremento de costos por incapacidades, estrés del personal o en el caso más grave por el desarrollo de enfermedad profesional asociada a la exposición persistente de niveles de ruido. ${ }_{30}$

En este contexto la literatura expone que en ocasiones en las unidades de cuidado intensivo se omite durante el diseño y remodelación la utilización de materiales que favorezcan la absorción de las ondas sonoras, en 
lugar de ello se encuentran superficies con poca o nula absorción acústica que contribuye a la generación de ruido y por ende, tienen implicaciones sobre el bienestar del personal, de los pacientes internados en las unidades de cuidado crítico y posiblemente sobre los costos dentro de la unidad. ${ }_{28}$

Teniendo en cuenta los niveles de ruido obtenidos en el presente estudio y las posibles implicaciones que puede tener a nivel económico, desde el punto de vista de salud del paciente y del personal asistencial que labora dentro de las unidades de cuidado crítico, es importante realizar seguimientos al nivel de ruido que permitan identificar y entender la dinámica acústica dentro del funcionamiento normal de las unidades de cuidado intensivo, y con ello adoptar programas y/o estrategias que contribuyan al bienestar fisiológico y psicológico de los pacientes y del personal que labora dentro de estas unidades.

En consecuencia sería interesante realizar un estudio indicando el porcentaje de eficiencia en la reducción de los niveles de ruido utilizando materiales absorbentes en el diseño de las unidades de cuidado crítico, y comprobar si es totalmente eficiente esta medida, o si por el contrario se requeriría la adopción de medidas adicionales para mantener los niveles dentro de los límites establecidos por organizaciones internacionales.

\section{Agradecimientos}

Los autores expresan sus agradecimientos al personal asistencial y administrativo de la unidad de cuidado intensivo adulto por su colaboración. De igual manera, se agradece a la Universidad del Magdalena y al Departamento Administrativo de Ciencia, Tecnología e Innovación - Colciencias por el apoyo a través del Programa Jóvenes Investigadores e Innovadores.

\section{Referencias}

1. Basco-Prado L, Fariñas-Rodríguez S, Hidalgo-Blanco MA. Características del sueño de los pacientes en una unidad de cuidados intensivos. Rev Cuba Enferm. junio de 2010;26(2):0-0.

2. Epp K. Burnout in critical care nurses: a literature review. Dynamics. 2012;23(4):25-31.

3. Christensen M. What knowledge do ICU nurses have with regard to the effects of noise exposure in the Intensive Care Unit? Intensive Crit Care Nurs. agosto de 2005;21(4):199-207.
4. Christensen M. Noise levels in a general intensive care unit: a descriptive study. Nurs Crit Care. 1 de julio de 2007;12(4):188-97.

5. Carvalho WB, Pedreira MLG, Aguiar MAL de. Noise level in a pediatric intensive care unit. J Pediatr (Rio J). diciembre de 2005;81(6):495-8.

6. Fernández DP, Cruz JN. Efectos del ruido en ambiente hospitalario neonatal. Cienc Trab. junio de 2006;8(20):65-73.

7. Alvarez AA, Terrón A, Boschi C, Gómez M. Review of noise in neonatal intensive care units - regional analysis. J Phys Conf Ser. 1 de noviembre de 2007;90(1):012038.

8. Drouot X, Cabello B, d'Ortho M-P, Brochard L. Sleep in the intensive care unit. Sleep Med Rev. octubre de 2008;12(5):391-403.

9. Chang TY, Lai YA, Hsieh HH, Lai JS, Liu CS. Effects of environmental noise exposure on ambulatory blood pressure in young adults. Environ Res. octubre de 2009;109(7):900-5.

10. Altuncu E, Akman I, Kulekci S, Akdas F, Bilgen H, Ozek E. Noise levels in neonatal intensive care unit and use of sound absorbing panel in the isolette. Int J Pediatr Otorhinolaryngol. 2009;73(7):951-3.

11. Kamdar BB, King LM, Collop NA, Sakamuri S, Colantuoni E, Neufeld KJ, et al. The effect of a quality improvement intervention on perceived sleep quality and cognition in a medical ICU. Crit Care Med. marzo de 2013;41(3):800-9.

12. Garrido-Galindo AP, Camargo-Caicedo Y, Vélez-Pereira AM. Nivel continuo equivalente de ruido en la unidad de cuidado intensivo neonatal asociado al síndrome de burnout. Enferm Intensiva. 2015;26(3):92-100.

13. EPA USO of NA and Information on levels of environmental noise requisite to protect public health and welfare with an adequate margin of safety for sale by the Supt. of Docs., U.S. Govt. Print. Off.; 1974. 196 p.

14. American College of Critical Care Medicine, Society of Critical Care Medicine. Guidelines for intensive care unit design. Crit Care Med. 1995;23(3):582-8.

15. Berglund B, Lindvall T, Schwela DH, others. Guías para el ruido urbano. Cent Panam Ing Sanit Cienc Ambiente OPSCEPIS [Internet]. 1999 [citado 18 de mayo de 2015]; Disponible en: https://editorial.cda. ulpgc.es/ftp/Instalaciones2/ACUSTICA/ANEXO/1Comodidad/OMSalud-Guias $\% 2$ para $\% 20$ el $\% 20$ Ruido\%20Urbano.pdf

16. Konkani A, Oakley B. Noise in hospital intensive care units-a critical review of a critical topic. J Crit Care. octubre de 2012;27(5):522.e1-522.e9

17. Guidelines/Practice Parameters Committee of the, American College of Critical Care Medicine, Society of 
Critical Care Medicine. Guidelines for intensive care unit design. Crit Care Med. 1995;23(3):582-8

18. Ministerio de Salud de Colombia. Resolucion 8321 de 1983, Agosto 4, por la cual se dictan normas sobre proteccion y conservacion de la audicion de la salud y el bienestar de las personas por causa de la produccion y emision de ruidos. Resolución 1983.

19. República de Colombia. Ministerio de Ambiente, Vivienda y Desarrollo Territorial. Resolución 627 de 2006 por la cual se establece la norma nacional de emisión de ruido y ruido ambiental. Resolución 627 de 2006: 29

20. Elliott R, McKinley SM, Eager DM. A pilot study of sound levels in an australian adult general intensive care unit. Noise Health. enero de 2010;12:26-36

21. Colina LM, Freites A, Gutiérrez P. Efectos no auditivos que ocasiona el ruido al personal de enfermería que labora en la unidad de cuidados intensivos de adultos (UCIA) del Hospital Central de Maracay. [Internet] [Tesis para optar el titulo de Licenciado en Enfermería]. [Venezuela]: Universidad Central de Venezuela; 2000. Disponible en: http://saber.ucv.ve/ jspui/handle/123456789/408

22. Macedo ISC, Mateus DC, Costa EDMGC, Asprino ACL, Lourenço EA. Noise assessment in intensive care units. Braz J Otorhinolaryngol. 2009;75(6):844-6

23. Vélez-Pereira AM, Camargo Caicedo Y. Análisis de los factores ambientales y ocupacionales en la concentración de aerobacterias en unidades de cuidado intensivo del Hospital Universitario Fernando Troconis, 2009 Santa Marta - Colombia. Rev Cuid. 2014;5(1):595-605.

24. Vélez-Pereira AM. Evaluación de niveles de ruido en la unidad de cuidados intensivos neonatal del Hospital Juan Ramón Jiménez [Tesis de Fin de Master, Master en Tecnología Ambiental y Gestión del Agua]. [Huelva, España]: Universidad Internacional de Andalucía Universidad de Huelva; 2010.

25. Fortes-Garrido JC, Velez-Pereira AM, Gázquez M, Hidalgo-Hidalgo M, Bolívar JP. The characterization of noise levels in a neonatal intensive care unit and the implications for noise management. J Environ Health Sci Eng. 22 de julio de 2014;12(1):104.

26. Berg AL, Chavez CT, Serpanos YC. Monitoring noise levels in a tertiary neonatal intensive care unit. CICSD. 2010;37:69-72.

27. Fajardo DL, Gallego SY, Argote LÁ. Noise levels in the Hospital Universitario del Valle newborn intensive care unit "Cirena», Cali, Colombia. Colomb Médica. 2007;38(4 Supl 2):64-71.
28. Johansson L, Bergbom I, Waye KP, Ryherd E, Lindahl B. The sound environment in an ICU patient room-A content analysis of sound levels and patient experiences. Intensive Crit Care Nurs. octubre de 2012;28(5):269-79.

29. Ayllón-Garrido N, Álvarez-González M, González-García M. Factores ambientales estresantes percibidos por los pacientes de una unidad de cuidados intensivos. Enferm Intensiva. diciembre de 2007;18(4):159-67.

30. Martínez-Suárez P, Moreno-Jiménez A. El ambiente acústico de los hospitales de Madrid: metodología de análisis y diagnóstico con SIG. Cuad Geográficos Univ Granada. 2006;(39):125-46.

31. Achury-Saldaña DM, Achury LF. Sueño en el paciente crítico: una necesidad insatisfecha en la unidad de cuidado intensivo. Investig En Enferm Imagen Desarro. 19 de agosto de 2011;12(1):25-42. 